\title{
DECORRELATING CLUTTER STATISTICS FOR LONG INTEGRATION TIME SAR IMAGING
}

\author{
Leanza Antonio $^{(1)}$, Monti Guarnieri Andrea ${ }^{(1)}$, Recchia Andrea ${ }^{(1)}$, Broquetas Ibars Antoni ${ }^{(2)}$, Ruiz Rodon Josep \\ (2) \\ (1) Dipartimento di Elettronica, Informazione e Bioingegneria - Politecnico di Milano, Via Ponzio 34/5, 20133 Milan \\ (Italy), antonio.leanza@polimi.it,monti@elet.polimi.it, andrea.recchia@polimi.it \\ (2) Departament de Teoria del Senyal i Comunicacions - Universitat Politecnica de Catalunya, Campus Nord D3 carrer \\ Jordi Girona 1-3, 08034 Barcelona (Spain), broquetas@tsc.upc.edu, josep.ruiz@tsc.upc.edu
}

\begin{abstract}
It is presented an experiment aimed to assess and eventually complement the Billingsley Internal Clutter Motion (ICM) model for long integration time SAR imaging. Exploiting a real-aperture rotating antenna Ground-Based RADAR, observations of rural areas in different periods of the year have been performed. The collected data, obtained from two different acquisition modes, have been processed to obtain short-term and long-term clutter de-correlation analysis. The results obtained revealed interesting aspects of the phenomenon. In particular, it can be observed that the process is nonstationary with time, say minutes to hours, and that DC/AC ratio follows a day/night characteristic. Moreover, the results showed values of the AC component decay rate $\beta$ higher than the foreseen ones in the considered spectral interval, which is quite below the one analyzed in the Billingsley experiment.
\end{abstract}

\section{INTRODUCTION}

The impact of clutter de-correlation on SAR imaging is negligible in the greater part of the applications and hence seldom mentioned. However, it becomes a relevant issue for some systems such as Moving Target Indication (MTI) RADAR or in the presence of particular targets, like for instance water surfaces [1]. Clutter de-correlation has to be carefully considered also in the design of all that systems that require medium or long integration time [2]. It is the case for example, of geosynchronous SAR or some cases of ground-based SAR [3]. Extensive studies on the matter have been carried out by Billingsley, who introduced a parametric Internal Clutter Motion (ICM) model [4]. This model has been widely validated for different wind speeds and frequencies. According to this model the clutter power spectrum $P_{t o t}(f)$ can be expressed as sum of a stable DC component $\delta(f)$ and a time-varying $\mathrm{AC}$ component $U(f)$ :

$$
P_{t o t}(f)=\frac{r}{r+1} \delta(f)+\frac{1}{r+1} U(f)
$$

where $r$ is the power ratio between the two components (DC/AC) and the AC component has the expression given by Eq. 2

$$
U(f)=\frac{\beta \lambda}{4} \exp \left(-\frac{\beta \lambda}{2}|f|\right)
$$

where $\beta$ is the parameter representing the decay rate. Different moving target classes are characterized by different values of the parameters $r$ and $\beta$.

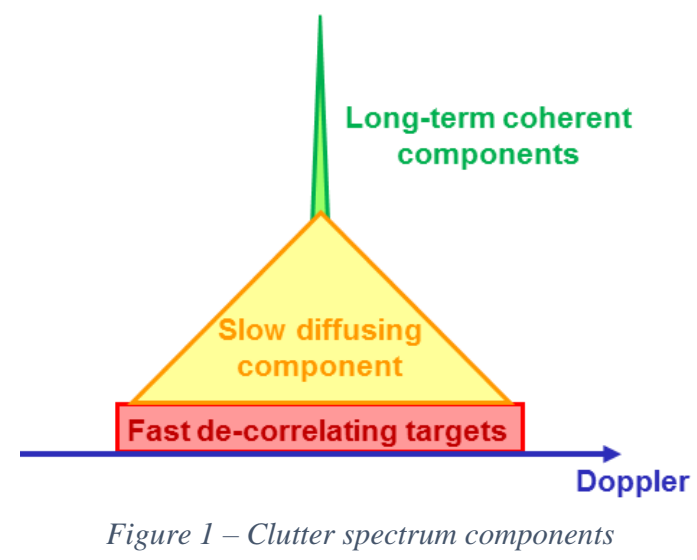

With reference to Figure 1 and considering a long integration time system framework, the harmful component of the clutter spectrum is the one that diffuses slowly along the Doppler. In fact, when the image is focused, the fast de-correlating clutter with Doppler higher than the azimuth bandwidth is spread outside the focused region. The long-term coherent component energy stays confined in the resolution cell, or at worst, in the neighbouring cells. The slow diffusing spectrum component in the azimuth bandwidth instead spreads his energy all over the image, producing a nuisance that may severely worsen the quality of the image or even jeopardize it. Clutter de-correlation is thus a critical aspect in the long integration time system and its impact has to be taken into account in the design.

\section{EXPERIMENT DESCRIPTION}

By means of a rotating real-aperture antenna GroundBased RADAR, a series of observation campaigns (see Table 3) of rural areas have been carried out. The selected scenarios are located in Switzerland and have been 
chosen in order to comprise different moving target classes (i.e. forest, crop) and stable targets (i.e. rocks, urban areas). All the campaigns took place under the low wind-speed condition. Since the experiment was aimed to investigate both short-term and long-term clutter behaviour, two acquisition modes were adopted and performed according to an interleaving scheme.
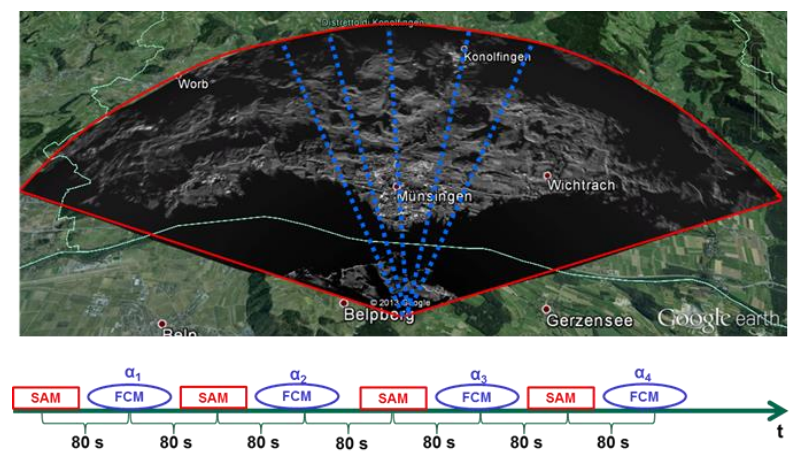

Figure 2 - Experiment acquisition modes and interleaving scheme

These two modes have been defined as

- $\quad$ Fixed Clutter Mode (FCM): The antenna is kept fixed at a given looking direction for the observation time and then is pointed toward a new direction for the next acquisition

- Scanning Atmosphere Mode (SAM): The target area is scanned repeatedly with a quick angular velocity and a short temporal baseline

In Figure 2 is shown a schematic representation of these two modes along with the interleaving scheme and Table 1 and Table 2 summarize their parameters.

Table 1 - FCM mode parameters

\begin{tabular}{lc}
\hline \multicolumn{1}{c}{ Parameter } & Value \\
\hline \hline Band & $\mathrm{Ku}$ \\
PRF [Hz] & 25 \\
Observation time [s] & 50 \\
Azimuth span [deg] & 70 \\
Azimuth sampling step [deg] & 2 \\
Range interval [m] & $50 \div 8440$ \\
\hline
\end{tabular}

Table 2-SAM mode parameters

\begin{tabular}{lc}
\hline \hline \multicolumn{1}{c}{ Parameter } & Value \\
\hline \hline Band & $\mathrm{Ku}$ \\
PRF [Hz] & 50 \\
Scan time [s] & 27.9 \\
Revisit time [s] & 160 \\
Azimuth span [deg] & 140 \\
Range interval [m] & $50 \div 8440$ \\
\hline \hline
\end{tabular}

For each observation campaign, the RADAR has acquired for an entire day. Hence, a FCM dataset consists of $\mathrm{N}$ observations of the selected $N_{a}$ azimuth directions and a SAM dataset consists of 24 hours scene scans repeated with the specified revisit time.

Table 3 - Observation campaigns summary

\begin{tabular}{|l|c|c|c|}
\cline { 2 - 4 } \multicolumn{1}{c|}{} & Campaign 1 & Campaign 2 & Campaign 3 \\
\hline Site & Chutze $(\mathrm{CH})$ & Chutze $(\mathrm{CH})$ & Bré $(\mathrm{CH})$ \\
\hline Date & Nov $25^{\text {th }} 2013$ & May $21^{\text {st }} 2014$ & Sep $02^{\text {nd }} 2014$ \\
\hline Modes & FCM, SAM & FCM,SAM & FCM,SAM \\
\hline
\end{tabular}

\section{SHORT-TERM CLUTTER ANALYSIS}

In FCM mode, the targets were observed continuously for a fixed time interval. These observations have been exploited to obtain a characterization of the short-term clutter behaviour in terms of Billingsley ICM model parameters.

\subsection{Data processing}

The short-term clutter analysis mainly consists in the comparison between the actual power spectrums of the moving targets observed during the FCM acquisitions and the Billingsley ICM model. In Figure 3 is represented the algorithm followed to obtain the results shown in par. 3.2 .

After a preliminary analysis of the data to identify the actual targets and discard the pure noise signals, the target power spectrum was computed from the echoes time series.

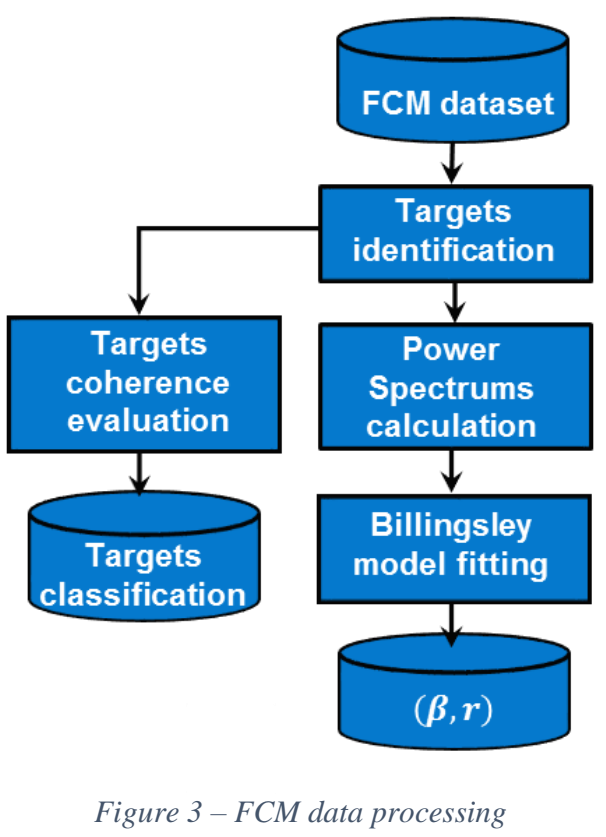

Simultaneously for each target, the coherence was calculated in order to classify them as Persistent Scatterer (PS) or Moving Target (MT). Finally a fitting operation between the actual spectrums and the Billingsley model was performed, determining the couples of parameters $(\beta, r)$, as shown in Figure 4. 


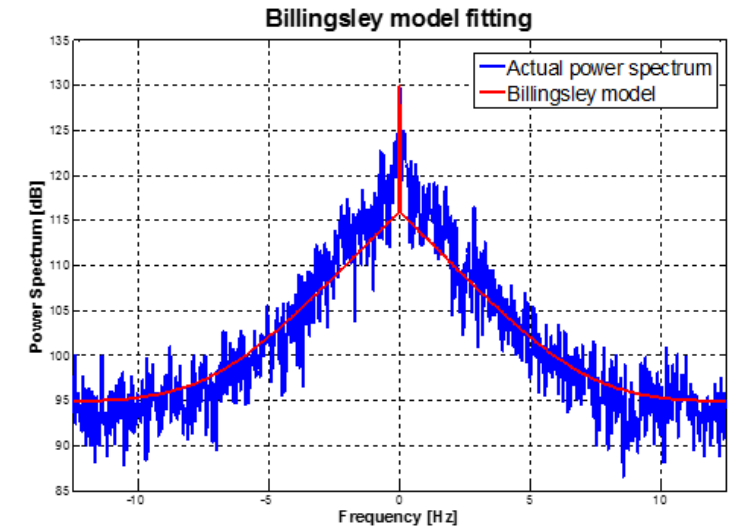

Figure 4-Billingsley ICM model fitting on the actual power spectrum of a moving target

\subsection{Short-term analysis results}

The fitting operation mentioned in par. 3.1 allowed the assessment of the Billingsley ICM model as clearly showed in Figure 4. Furthermore, it provided the estimation of the $(\beta, r)$ parameters. The results showed here in par. 3.2 and in par. 4.1 have been obtained considering only moving targets and with similar windspeed condition.
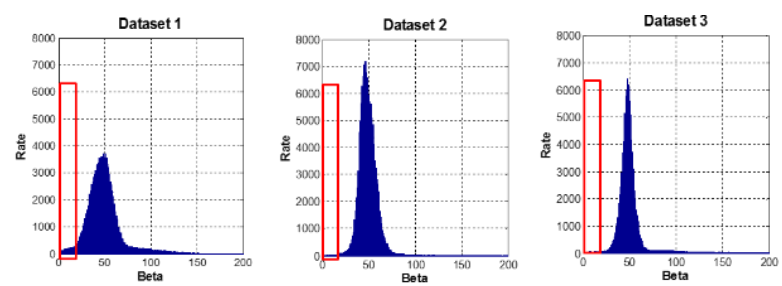

Figure $5-\beta$ values distribution for the 3 considered FCM datasets. In the red box the values interval foreseen by Billingsley for low wind velocity

With reference to Figure 5, a first interesting aspect about $\beta$ parameter can be noticed. In all the considered campaigns, the $\beta$ values observed are higher than the ones foreseen by Billingsley. In fact, Billingsley foresees values in the interval $4 \div 12$ for a low wind-speed situation, say from 0 to $10 \mathrm{~m} / \mathrm{s}$. This is compatible with Billingsley model since the present experiment considered a spectral interval quite below the one considered by Billingsley (short acquisition times with PRF of hundreds of $\mathrm{Hz}$ ).
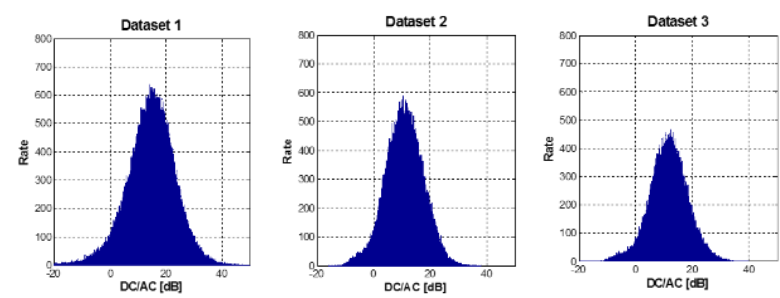

Figure 6-DC/AC ratio values distribution for the 3 considered FCM datasets.

Another interesting aspect is the different behaviour of the clutter between the first and the second campaign, which took place in the same site but in the different periods of the year. In fact, the first was realized at the end of November and the second at the end of May. As appears clearly in Figure 7 and by the associated histograms in Figure 5, the first campaign presents more dispersed $\beta$ values than the second one.
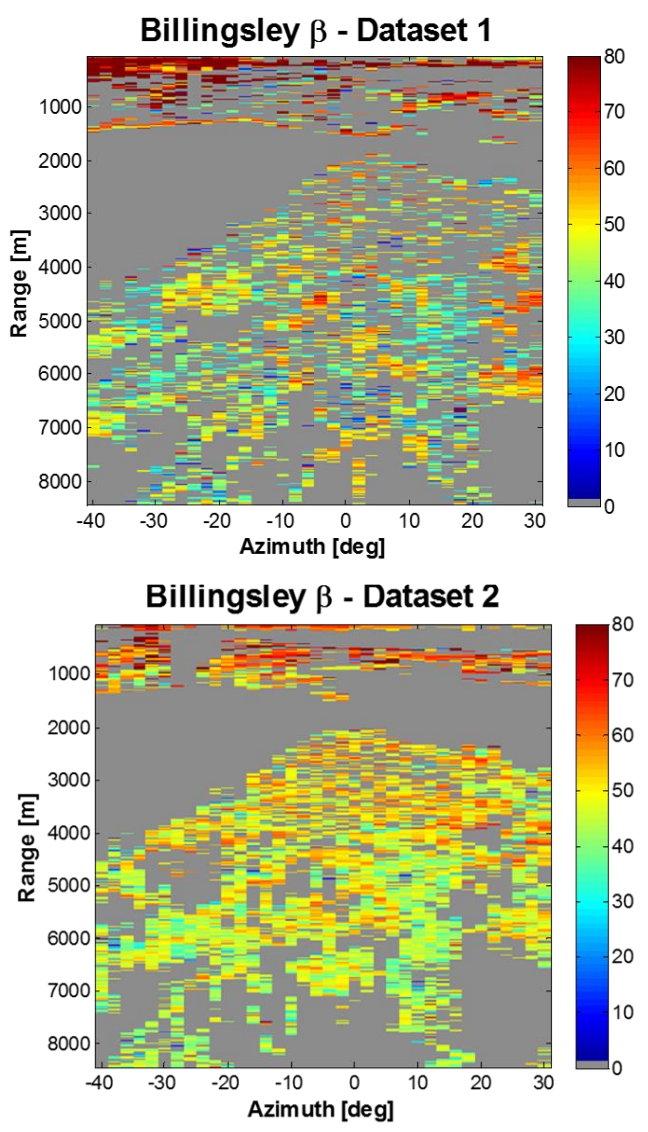

Figure $7-\beta$ values measured on the same location (Chutze) in two different periods of the year: late fall (up) and late spring (down)

The difference between the first campaign and the other two is also given by the other Billingsley parameter. In Figure 6 can be noticed that DC/AC values result higher in the first campaign. This different behaviour in terms of DC/AC of the first campaign can be noticed also in Figure 8 that shows the comparison with the third campaign. These differences would indicate a dependence of the clutter on the considered period of the year. Anyway, this dependence needs to be furtherly investigated and assessed by means of other observations.

\section{LONG-TERM CLUTTER ANALYSIS}

Long-Term analysis was carried out exploiting SAM datasets. Since this analysis focused on a very narrow bandwidth around the DC component (fraction of $\mathrm{Hz}$ ), only DC/AC parameter was considered. In this analysis, we assumed the stable DC component as the portion of 
the power spectrum in the bandwidth $B \leq 1 / T_{0}$ where $T_{0}=20 \mathrm{~min}$.
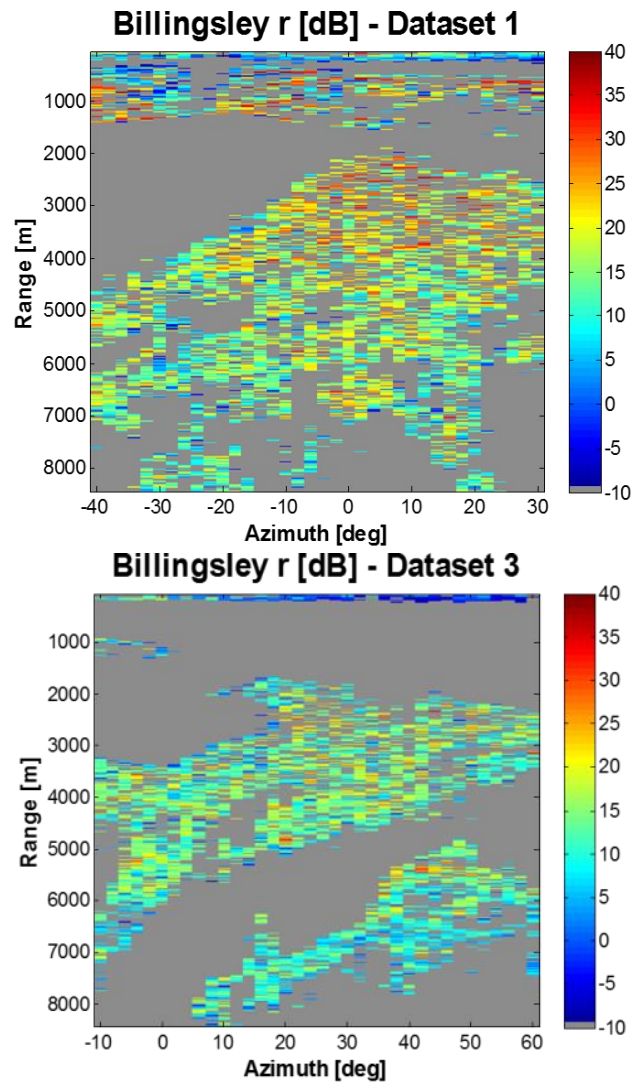

Figure 8-DC/AC values comparison between the autumn campaign (up) and the summer campaign (down)

\subsection{Data processing}

Long-Term analysis consists in the measurement of moving target stability in a time interval of several hour. In such a context, the assumption of constant Atmosphere Phase Screen (APS), adopted in the short-term analysis, is no longer valid. Thus, the APS contribution was preliminarily estimated and compensated. With reference to Figure 9, the APS was estimated exploiting the interferometric phase of the PS target. These targets were identified by the evaluation of their coherence. Finally, the moving targets DC/AC values were calculated from the APS compensated SAM images. As shown in par. 4.2 , clutter is not a stationary process, so the DC/AC was evaluated on two hours SAM sub-stacks.

\subsection{Long-term analysis results}

Moving targets de-correlation in time intervals of several hours was evaluated for the three campaigns considered. Since this process is non-stationary, evaluating the decorrelation on the whole SAM datasets (24 hours) is meaningless, hence the analysis was carried out evaluating the DC/AC from two hours data sub-stacks.

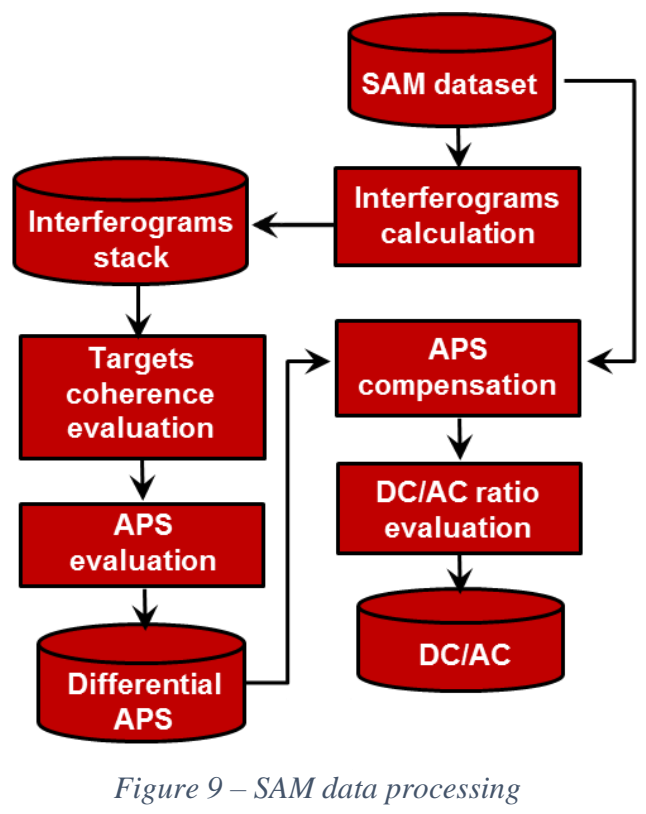

This aspect of the investigated problem is clear observing the coherence of the scene evaluated on the entire dataset shown in Figure 10. In fact, the coherence is related to the DC/AC by the Eq. 3

$$
\gamma=\frac{\gamma_{0}}{1+r^{-1}}
$$

where $\gamma_{0}$ is the de-correlation free coherence and $r$ represents the DC/AC. In Figure 10 is also shown (red box on the colorbar) the values of DC/AC foreseen by Billingsley ICM model for low wind-speed, which is our case for all the considered campaigns.

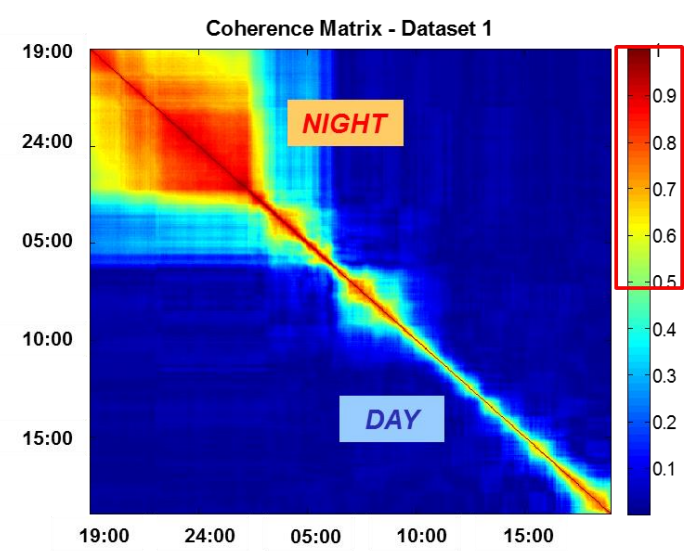

Figure 10-Scene coherence of the entire SAM dataset of the first observation campaign (only moving targets)

The analysis of DC/AC highlighted interesting aspects of clutter de-correlation. Referring to Figure 11, the statistics of the DC/AC values indicate a dependence of this parameter from the hours of the day. Specifically, the mean value of $\mathrm{DC} / \mathrm{AC}$ increases during the hours from 
dusk to dawn for all the campaigns, thus the targets result more stable during the night. This characteristic is also inferred by the scene coherence (see Figure 10). Furthermore, the DC/AC statistic in Figure 11 confirms the difference between the first and the other two campaigns, mentioned in the short-term analysis. In fact, $\mathrm{DC} / \mathrm{AC}$ mean values result higher in the first campaign, indicating a higher degree of stability of the scene.

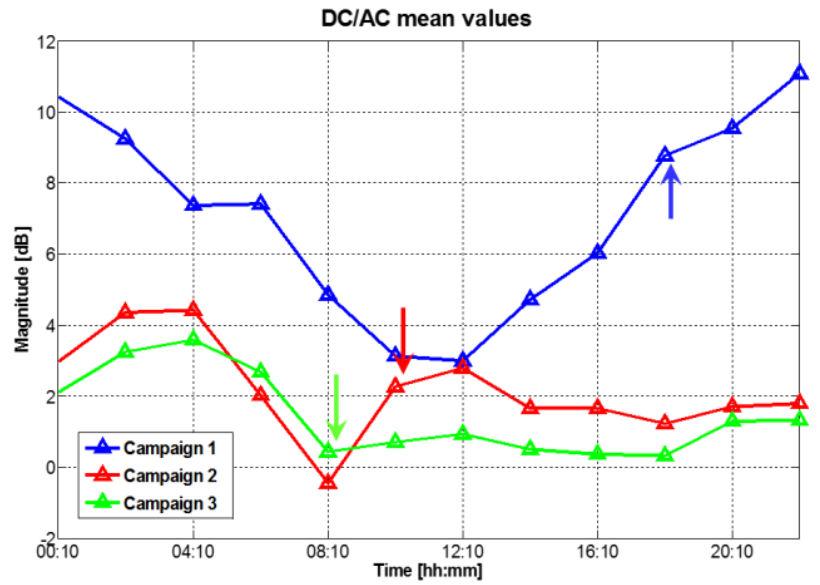

Figure $11-D C / A C$ mean trend of the 3 campaigns. Data is time circularly shifted and aligned to ease the comparison.

The actual starting times are indicated by the arrows.

\section{CONCLUSION}

In medium or long-integration time system design, clutter de-correlation cannot be neglected since it could affect massively the performances. This experiment was carried out with the objective of assessing the Billingsley ICM model in such a context and eventually complement it. The results showed that the validity of the ICM model still stands but with some differences. In particular, the values of the decay rate $\beta$ resulted higher than the values foreseen by Billingsley. An interesting difference emerged from the comparison of the first campaign with the other two consisting in a greater stability of the targets. This characteristic, confirmed by both the analysis performed, could indicate a relation between the phenomenon investigated and the period of the year and needs further investigation to be confirmed. Long-Term analysis showed how clutter de-correlation is a nonstationary process and has to be considered for time intervals not too long in the order of few hours. It also highlighted the day/night characteristic, observed in all the observations. This characteristic consists in an increasing value of the mean DC/AC in the hours from dusk to dawn, which implies a reduced targets decorrelation.

\section{REFERENCES}

1. Lombardo, P.; Greco, M.; Gini, F.; Farina, A.; Billingsley, J.B., "Impact of clutter spectra on radar performance prediction," Aerospace and Electronic
Systems, IEEE Transactions on, vol.37, no.3, pp.1022-1038, July 2001

2. Ruiz-Rodon, J.; Broquetas Ibars, A.; Makhoul, E.; Monti-Guarnieri, A.; Recchia, A., "Internal clutter motion impact on the long integration GEOSAR acquisition," Geoscience and Remote Sensing Symposium (IGARSS), 2014 IEEE International, pp.2343-2346, 13-18 July 2014

3. Recchia, A.; Monti Guarnieri, A.; Broquetas Ibars, A.; Ruiz Rodon, J., "Impact of clutter decorrelation on Geosynchronous SAR," EUSAR 2014; 10th European Conference on Synthetic Aperture Radar; Proceedings of , pp.1-4, 3-5 June 2014.

4. Billingsley J., "Low-angle Radar Land Clutter: Measurements and Empirical Models", ser. Radar, Sonar, Navigation and Avionics Bks. William Andrew Pub., 2002 